\title{
Applications of Fuzzy Technology in Business Intelligence
}

\author{
A. Meyer, H.-J. Zimmermann
}

\author{
Andreas Meyer \\ INFORM Institut f. Operations Research und Management GmbH \\ Risk \& Fraud Division \\ Pascalstr. 23 \\ 52076 Aachen, Germany E-mail: andreas.meyer@inform-software.com

\section{Hans-Jürgen Zimmermann} \\ RWTH, Aachen Institute of Technology \\ Templer Graben 55 \\ 52062 Aachen, Germany \\ E-mail: zi@or.rwth-aachen.de
}

\begin{abstract}
Fuzzy Set Theory has been developed during the last decades to a demanding mathematical theory. There exist more than 50,000 publications in this area by now. Unluckily the number of reports on applications of fuzzy technology has become very scarce. The reasons for that are manifold: Real applications are normally not single-method-applications but rather complex combinations of different techniques, which are not suited for a publication in a journal. Sometimes considerations of competition my play a role, and sometimes the theoretical core of an application is not suited for publication. In this paper we shall focus on applications of fuzzy technology on real problems in business management. Two versions of fuzzy technology will be used: Fuzzy Knowledge based systems and fuzzy clustering. It is assumed that the reader is familiar with basic fuzzy set theory and the goal of the paper is, to show that the potential of applying fuzzy technology in management is still very large and hardly exploited so far.
\end{abstract}

Keywords: fuzzy technology in business intelligence, fraud detection, risk assessment, intelligent data mining, fuzzy expert systems

\section{Introduction}

In his first paper on Fuzzy Sets L.A. Zadeh [20] already mentioned as one motivation of his Theory of Fuzzy Sets: "The fact remains that such imprecisely defined 'classes' play an important role in human thinking, particularly in the domains of pattern recognition, communication of information, and abstraction." This statement has become even more true in the meantime since we have moved from a time of scarce data into the period of data warehouses etc., i.e., into a world of abundance of data, in which people try hard to extract useful information from masses of data ( [24]).

From the point of view of applications we still consider as some of the most important goals of fuzzy set theory to extract information from data and model it visually or otherwise in such a way, that people can understand it, communicate it and to model problems adequately. They can use it to solve their problems better than by a purely dichotomous modeling language. In doing this professionally, they can combine the high computing power of EDP with human experience and creativity.

Extremely successful fuzzy models were first used in engineering intelligence in areas such as Fuzzy Control ( $[13,24,26,30])$. Controlling cranes, cement kilns, video cameras, washing machines, ABS, and even subway systems by fuzzy control turned out to be almost sensational. Most 
of these applications had one feature in common: These were manmade systems, the control of which was often nonlinear (and therefore difficult to model traditionally). However the controls could be decomposed into linear systems by modeling human experience by fuzzy technology ( [27]) and one could then determine the adequate parameters, operators ( [6]) and membership functions, as well as the defuzzification models, by trial and error. Practitioners loved these controllers because they used predominantly rather basic fuzzy set theory and operators as well as membership functions could be defined rather than determined on the basis of human knowledge. Many of these models have become regular teaching material in control engineering courses.

In the meantime fuzzy set theory was further developed, it became more powerful mathematically and it became more strenuous to learn and understand it. When one started to apply it to business intelligence and to human decision making another problem became visible: Many applications do not permit a trial and error calibration as in fuzzy control because the results of a fuzzy model cannot easily be compared with the results or the behavior of the real system. Think of strategic decisions, of evaluations of long term vulnerability of companies or persons, the determination of the creditworthiness ( [29]) of persons or institutions. Here the human knowledge that goes into the fuzzy model has to be modeled properly in advance. That means, that operators ( [17]), membership functions, inference methods ( [27]) etc. have to properly map the counterparts in the human mind, in which they are very often very context dependent. This is no longer only a mathematical problem but predominantly a problem of psycho-linguistics or similar disciplines $([7,25])$. Unluckily this part of science is much less developed than the mathematics of fuzzy set theory. Hence, in applications one often has to rely again on assumptions rather than on scientific results when modeling operators ( $[19,28])$, membership functions $([5,9,16])$ and other parts of fuzzy models. The justification of assumptions, of course, also depends on whether one wants to build descriptive or prescriptive models.

In the following sections we want to show, that, never-the-less, practical applications of fuzzy models in the area of business intelligence are also possible and very useful.

\section{RiskShield - A Software Solution Relying on fuzzy technology}

This chapter describes the real world software application RiskShield [11] which has predominantly been developed in the years 1999 to 2010. RiskShield is one of the leading tools in the European payments/banking and insurance sector to fight fraud. Well-known air cargo organizations currently consider its possible use to enhance air freight security at acceptable costs. We will report on the practical use of RiskShield in the mentioned industries in Chapter 3.

To briefly describe the solution independently of its purpose and industry in which it is used (payments/banking, insurance, air cargo) we will describe the solution rather in the context of banking, i.e. the term 'transaction' may refer to a financial transaction in the payments sector, to a claim in the insurance sector or to a set of information concerning a parcel in the air cargo sector.

\subsection{Software Description}

\section{The Basics}

RiskShield assesses the risk of incoming transactions individually in real-time within milliseconds. The software works for the human user invisibly in the background. An IT application of the client (e.g. the bank or insurance company) sends every transaction to RiskShield. The software's multi channel approach includes real-time evaluation of additional statistical data and patterns. It builds up internal histories for all relevant entities in the process. Typically 
RiskShield installations include 10 to 20 profiles which are constantly updated with any incoming event. Predefined groups can be established to detect any untypical behavior. Fuzzy technology is used to evaluate all different aspects - in many cases the combination of current information with dynamic patterns and histories is needed to identify critical events.

For every incoming transaction RiskShield returns a risk score which triggers the possible decision for (manual) special treatment of the transaction. Depending on configuration the software product can further create a case (alarm) to be further manually investigated and returns the main indicators for the suspicion to bring the human investigators in a more comfortable position to start the special treatment of the transaction (see Figure 1). Every transaction is scored against the same RiskShield decision system to assure consistent results. To evaluate all information provided to the system several rules and patterns of the decision system may contribute to the assessment of an individual transaction.

The type of fuzzy inference used in RiskShield corresponds to the inference described for fuzzyTECH in [15], [30, pp. $255 \mathrm{ff}$.$] .$

\section{Other Logics and Techniques of RiskShield}

RiskShield utilizes several complementary techniques like fuzzy technology [10], matching of patterns against dynamic profiles, recognition of networks (see below) and external lists to ensure a good risk assessment quality. A combination of those techniques forms the decision system within the software solution to assess the risk of a single transaction, i.e. the threat potential which may be caused by the respective transaction.

Matching of patterns against dynamic profiles:

The decision making system can use several profiles which can be defined according to the requirements. Based on these profiles RiskShield recognizes defined patterns in real-time which can comfortably edited by in-house risk and fraud experts without internal IT support or support by the vendor INFORM ( [11]).

Recognition of networks and external lists:

Through RiskShield it is possible to detect social and historical relationships based on historical data as well as to use external lists. The meaning of an external list can be defined within the decision system. Therefore these external lists can be used in different ways, e.g. to define positive/white lists or negative/black lists. An example for a white list in Internet banking systems might be a list of account numbers or a list of beneficiary accounts; in credit card issuing systems a black list might consist of BIN-ranges (first $\mathrm{x}$ digits of the credit card number); in the air freight area a black list of addressees could be derived from a list of PEP (politically exposed persons) which also receive a special treatment in RiskShield applications for anti money laundering. External lists can not only form white or black lists but also any other list which is relevant for the decision making system. The entries of each list can be associated to a valid-until timestamp, if required.

The combination of these complementary techniques offers the RiskShield user exhaustive possibilities to build a complete safety net dealing with multi-channel fraud attacks and threats.

\section{Response and Alert Management}

The risk score RiskShield has returned is used for a decision and an automated workflow control (possibly special treatment of a transaction). This score represents the decision which 
has been made regarding the current transaction (Approval, Referral, Decline, Hold Movement/ Hold Payment).

Additional scores and information can be defined to be returned by the software solution, e.g.

- Case classes: Groupings of alerts regarding the class of the transaction or the assumed type of fraud.

- Importance: Alerts can be rated regarding their importance so that the most important alerts can be worked with first.

- Hints: A list of rules which had a main impact to the score of the transaction.

- Actions: Criteria can be defined to trigger consecutive actions, e.g. send email/fax/sms, update master data, provide information to CRM, get address scoring.

RiskShield offers the possibility to document the investigation process of the respective alert through a web front end. Additionally, the system offers further analysis functionality to monitor and trace each assessment in detail (drill-down functionality).

RiskShield's use of fuzzy inference combined with matching of patterns against dynamic profiles, recognition of social and historical relations and external lists provide superb fraud prevention qualities for the payments, insurance and probably also for the air cargo security sector. As a result users will obtain almost only relevant hits due to the low false positives output. Research has shown that the automated identification of suspicious transactions for further investigation matches senior analyst evaluations to a very high percentage of cases.

\section{Other Key Features of RiskShield}

- Real-time decision making

- Response and other actions within milliseconds

- Up to 4,100 single assessments per second

- Processing

- Automatic threat hit matching

- Analysis and simulation

- Analysis and complex simulation with production data (chart, table, and record filtering)

- All decisions and decision criteria are transparent to the threat analyst

- Implementation

- Standard interfaces (XML, CSV, JMS)

- Pre- and Post-Processing plug-ins for quick implementation

- Software Updates within minutes

- external support not needed

- backward compatibility

- Supports a high secure PCI-DSS compliant implementation (relevant to credit card business) 


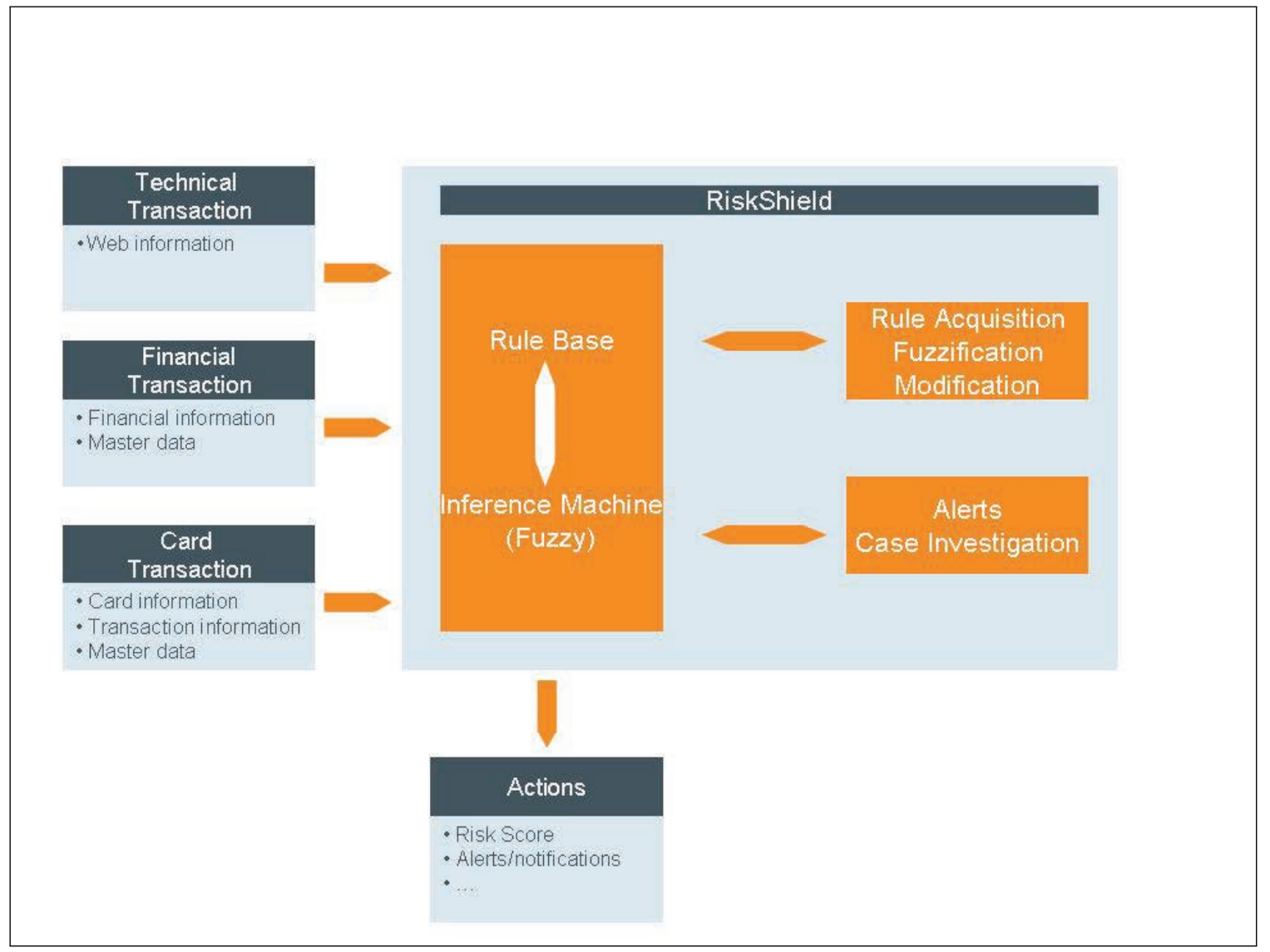

Figure 1: Scheme of RiskShield (input data exemplarily from payments sector)

\subsection{Benefits of Fuzzy Logic from Real World Users' Perspective}

Real world business users from the industries banking/payments, insurance and air cargo perceive that fuzzy technology as a basis of automated software solutions gives a competitive edge for the following reasons:

- Decision systems using fuzzy logic represent experience of experts adequately:

Fuzzy logic lets you rely on the experience of people who already understand your system.

- Fuzzy logic is flexible:

With any given system, it is easy to layer on more functionality without starting again from scratch.

- Fuzzy rules consider fraud/threat and non-fraud/non-threat indicators:

Like a human expert all aspects of decision making can be integrated into a decision system using fuzzy rules.

- Decision systems using fuzzy logic are easier and more cost-effectively to maintain:

Before changing an existing rule or adding a new one the impact of the changes to the overall decision system has to be analyzed. As fuzzy logic systems require much less rules than conventional rule based systems the impact of possible changes can be anticipated much easier. Decision systems using fuzzy logic result in shorter reaction times to new threat patterns As explained new rules can be set into productive use faster. 
- Fuzzy logic helps minimize false-positives:

The concept of fuzzy logic represents fuzzy bounds. Thus, the usage of hard bounds which typically result in false-positives is obsolete.

- Decision systems using fuzzy logic are more reliable:

Conventional rule bases systems have to consider a lot of special cases. One missing case may lead to completely unexpected behavior of the system. Within fuzzy systems the differentiation between special cases is not needed so much.

\section{Fraud Prevention and Risk Assessment}

In this chapter we report on the practical use of fuzzy logic through the software product RiskShield (see the description in Chapter 2) to prevent fraud in the payments industry (Section 3.1), to detect insurance fraud (Section 3.2). Furthermore we describe its possible use to enhance air freight security at acceptable costs (Section 3.3).

\subsection{Payments Industry}

\section{The Challenge}

In the last few years, fraudsters have become well organized and well equipped to work professionally to attack the payments industry. They prefer to attack payment systems with a low ratio of effort/success. This is in particular the case for online banking platforms where it is possible to use automated scripts like Trojans and viruses that capture the customer online banking session to communicate directly with the attacking server platform (man-in-the-middle and man-in-the-browser attack). Furthermore, phishing attacks are popular as they require not much manual effort.

In the past hardware based IT security measurements have been sufficient for many years to protect Internet banking transactions against fraud, as fraud attacks followed relatively simple patterns. This has changed completely in the last few years. Hardware based IT security products used by end-customers are static regarding its security functionality. That means once they have been issued they won't change their behavior. So when it turns out in practice that the hardware products are not resistant to some online attack banks had to implement an additional security measurement or to replace at high overall costs the hardware products for all customers. Today even banks with the best equipped platforms realize, that relying solely on hardware based protection is too risky (as reaction to new fraud patterns takes too much time in practice) and too expensive.

\section{The Patterns}

Different data is required to identify a fraud pattern and protect the account. We need to combine all information we have available in the environment as there are many questions to be answered.

What is the usual type and nature of the customer's transactions? Which beneficiary accounts is he using and what do we know about it? Does the amount of the current transaction exceed the usual payments done by this customer?

Are there other account holders for this account and does this transaction fit to one of their profiles? The transaction might be not typical for the account holder, but may be it is typical for the beneficiary account? What other products does the customer have and how does he use them? Is he a share holder? How many cards does he have?

What do we know concerning the IP address? Is the IP address well known in combination with 
this customer? Or is a foreign IP address unexpected for this customer that is always using one of the domestic providers?

What happens during the internet session? Does the IP address change suddenly? Is the usage of the online banking web application as expected?

\section{The Solution}

The approach to effectively fight against the current sophisticated attacks should be a combination of expert knowledge ( [26]) and a generic software solution. The technical expert must have knowledge of the design, workflow, and architecture of the existing online banking platform and the business expert must have knowledge about the bank's products and customer profiles.

Fortunately, fraud patterns have fingerprints that help to identify them. Once the pattern is identified, a software solution is required that is capable to process all required data sources, to combine the information streams, to identify the sequence of events, to be enhanced on-the-fly, and that is connected to the existing IT environment to stop the payment and to generate alerts. Fuzzy technology ( [21-23]) can easily model expert knowledge about fraud patterns and put it into a software solution. For example, a rule like the following could cross the mind of a human expert and could easily be entered into a fuzzy logic based solution like RiskShield:

In case of a payment from a customer account with an amount of untypical high value, an IP address from a risky region instead of the well known IP address for this account, stop payment for manual intervention.

An effective ant-fraud solution has to address further important issues:

Noise Reduction: An online banking fraud prevention software solution would be useless, if the quality of the generated alerts would be so bad that it led to an inflation of alerts which hide the single valid fraud alarm. So "noise" reduction is required to get the most important alerts only. As RiskShield implementations have shown, this is possible by a most powerful anti fraud pattern definition that filters out undesired alerts down to an acceptable false-positive rate. When evaluating measurable input data RiskShield uses fuzzy bounds instead of hard bounds to minimize false-positives. Particularly in this regard, fuzzy logic is an important underlying concept for the system.

Alerts: Once the payment has been put to hold by RiskShield the case investigation user has time to access the alert data, to verify all the data displayed, to switch the view from the account layer into the customer or beneficiary layer, and to review the profiles and statistical data. This helps to get an overview about the situation in total.

Actions: After investigating the case, the user has the possibility to initiate further actions like releasing or finally stopping the payment by one mouse click. Other actions can be attached to the workflow as well, like an online update of the RiskShield-Server to have this information available for all further decisions. Once all necessary actions have been initiated, the user closes the case which is then sent to the RiskShield reports to check the efficiency of the case queues and users.

Regarding the use of fuzzy technology RiskShield supports its users e.g. through graphical editors to define membership functions, to determine operators, to automatically perform the inference process and to determine a suitable defuzzyfication process.

In the payments industry RiskShield is also in use to detect fraud in other areas $([4,8])$ (so-called channels) including: debit cards and credit cards from the perspective of the issuing bank or from the merchant's or acquirer's perspective, ATM skimming, loan application and others. The software product's multi-channel approach combines findings about a customer across different channels to better assess whether a current transaction from a specific channel 
has indeed been authorized by the supposed customer.

\subsection{Insurance Industry}

Due to insurance fraud insurance companies across the globe lose significant amounts of money. The sum of total payments to fraudsters may even by far exceed the profits generated by an insurance company. Insurance experts assess that, depending on the line of business and country, more than 10 percent of all claims notifications have a fraudulent background.

Insurance companies are nowadays battling strongly to keep customer's affections. However, they perceive too high fraud losses to be unacceptable. Regarding their claims handling process insurance companies are typically faced with the challenge to reduce settlement and handling costs of claims on the one hand and to increase customer satisfaction on the other hand. Increase of customer satisfaction includes low insurance premiums and fast, unbureaucratic settlement of legitimate claims. Reduction of settlement and handling costs seems to be a contradictory objective. Because due to the typically high percentage of fraudulent claims, it seems insurance companies have to manually review each single claim very carefully. But this would increase the claims handling costs and would annoy the honest customers awaiting fast settlement of their legitimate claim.

Recently we have recognized the trend among the rather big insurance companies to look for the introduction of some kind of intelligent software which ensures that simultaneously legitimate claims are assigned to a fast settlement track and suspicious claims are assigned to senior fraud experts of the company for further manual review. To do so, the software should automatically assess each single claim and make a decision on how to proceed with the claim. The outputs of the software have to be transparent and traceable. Claims handlers and fraud experts of the insurance company may rather accept a fraud detection software in case they feel confident the software assesses the claims in the same way they would do. That is why expert systems based on fuzzy technology, e.g. RiskShield from INFORM, seem to be well suited to assess insurance claims. In doing so, RiskShield takes both incriminating and exonerating indicators into account. The system balances these indicators as a human expert would do and finally consolidates them to an overall risk score. Expert rules are formulated in a linguistic if-then context, similar to the human language. Furthermore, RiskShield gives additional outputs to indicate the reason of the assessment or to advice what to do next in terms of claims handling. Moreover, RiskShield checks, as a human claims handler would do, for each incoming claim whether the claim related information is already sufficient for a qualified evaluation.

The process of gauging like a human expert when evaluating a claim is illustrated in the following example:

- A new claim, let's see...

- A new contract ... (incriminating)

- A 2,000 claim, thus a minor loss of property ... (exonerating)

- A brand new Mercedes ... (incriminating)

- No police on scene ... (incriminating)

- The car will be repaired at a partner workshop ... (exonerating)

- A minor damage with no injuries ... (exonerating)

- The policy holder has had only few previous claims ...(exonerating) 
- It is a long time customer re other lines of business ... (exonerating)

- Conclusion: I think we should pay this claim.

INFORM typically delivers its product RiskShield accompanied by consulting services. These services include the provision of customized fraud detection and claims assessment logics which turned out to combine superb fraud detection quality with minimum false positives: Claims marked by RiskShield as suspicious for further investigation match senior analyst evaluations typically in more than 90RiskShield's fraud detection rules consider different types of information like the policy holder, the claims history, the policy itself, the policy history, the loss, and others. Fuzzy technology is useful in the given context to reflect human thinking and to use soft boundaries instead of crisp boundaries which in general will be easily explored and misused by fraudsters.

An insurance claim automatically classified by RiskShield as inconspicuous can be settled faster and more cost-effectively and so strengthening customer loyalty. The resulting saving in time at claims handlers and senior fraud experts for a detailed investigation of claims which have been classified as unusual leads to the reduction of fraud losses which can save a insurance company millions every year.

The fuzzy technology based solution RiskShield is successfully used in more than 35 insurance portfolios across Europe and North America for automatic claim evaluation and fraud risk assessment. Recently the software solution has also been applied to detect medical billing fraud. In a pilot scheme for one of the leading US car insurance companies INFORM analyzed through RiskShield four years of data of all medical billing claims submitted to the insurance company in one US state. INFORM figured out that approximately $18 \%$ of all medical bills are to a significant extent incorrect resp. fraudulent.

\subsection{Air Freight Security}

The latest terror attack attempts using explosive parcels in air freight result in the need to increase security through extended security controls of the parcels at affordable additional costs. A manual reliable security check of every parcel to be delivered cannot be implemented in practice from both organizational and costs perspectives. This subsection describes the idea of applying software-based monitoring solutions which have been proven in the payments/banking sector to fight fraud and to detect irregular patterns, to the air freight sector to identify in realtime suspicious parcels to be delivered. It is reasonable to assume that an air cargo organization already has the required information like sender, addressee, place of posting, freight declaration and other specifications electronically available and possibly stored in one central or a few central applications or data bases. The combination of such pieces of information plus some specific knowledge of the typical behavior of geographical regions as senders (typical previous movements) and the parcels an addressee typically receives should be sufficient from our view to identify suspicious items of freight. To fully automate this process air cargo organizations may consider the use of INFORM's software RiskShield which relies on fuzzy logic as an underlying core technology to keep false alarms acceptably low. Items which have been identified by RiskShield as suspicious will in the air cargo scenario be referred for manual investigation. Air cargo companies currently consider the use of the software solution as it allows economically justifiable close checks to increase airfreight security to a much higher level. In case an air cargo organization already have implemented comprehensive X-ray checks, possibly for every single parcel due to regulatory provisions like from Luftfahrtbundesamt in Germany, a software like RiskShield would 
nevertheless increase the security level as in practice one possibly cannot trust in the reliability of a single X-ray check, especially if such X-ray checks have been taken abroad in a "risky country".

\section{Customer Segmentation in Financial Services}

\subsection{Crisp vs. Fuzzy Clustering}

Banks have different products to offer to their customers ( [14, pp. 3-9]), they have (very) different customers for which the products are differently suited, and they have a lot of information (data) of their customers. An efficient marketing of these products requires, as in other markets, a segmentation of the customers such that only suitable products are offered to the appropriate class of customers. Even to day often this segmentation is performed by using single criteria such as age, property, income etc. This obviously does not lead to adequate classes of customers. A better way to classify customers is to use classical clustering, in which a number of attributes can be used to describe a customer. This, however, leaves the problem of objects (e.g. customers) that can not be assigned unequivocally to any particular cluster. With fuzzy cluster analysis ( [18]), such as the fuzzy c-means algorithm $([2,3])$, each customer is not assigned to one and only one cluster but rather with different degrees of membership to each class or segment. For outlyers the degrees of membership might not be meaningful, but the use of possibilistic clustering ( [12]) cures even this weakness by normalizing the sum of all assigned degrees of membership for each element (customer) to be 1 .

\subsection{Segmenting Customers of Banks}

Let us consider a (smaller) data base of 120,000 records of customers, from which 300 records were samples randomly ( $[14$, p. 3]). The sample records contain all the data fields which are available via the bank's customer information system, such as: age, sex, marital status, number of children, income, assets, information on other banking activities and also the bank's profit margin for each individual customer. The next step would be to select those features from all the characteristic describing a customer, which are relevant for customer segmentation. After discussions with experts the following features may be relevant:

- age (in years)

- net monthly income

- financial assets deposited with the bank

- loans taken out from the bank

- annual marginal gain for the bank. This value denotes the sum which the bank earns or loses from the individual customer per year.

Now the optimal number of clusters has to be determined. A number of criteria have to be satisfied: It should be possible to interpret the different clusters in terms of the expert's vocabulary; the classification should make sense in terms of the different products the bank wants to sell, etc. Technically two measures vary with the number of clusters: the partition coefficient (increasing with the number of clusters) and the partition entropy (falling with the number of clusters). Both tend towards monotone behavior depending on the number of clusters. As the optimal number of clusters can be considered the number where the entropy lies below the rising trend and where the value of the partition coefficient lies above the falling trend. The "optimal" number of clusters is determined by simulation. In our case the best number of clusters is 7 and 
the cluster centers are as shown in figure 2 (from [14, p. 16]). In this table the clusters have already been "labeled" by experts.

Figure 3 (from [14, p. 17]) shows the membership values of the cluster centers. It is obvious, that a crisp clustering would never have arrived at such a classification. The rest of the customers can now be classified according to the 7 classes. This can be done directly by using DataEngine or normally by the respective c-means algorithm in the software system of the bank. The goal of such a classification run can either be to segment customers in a databank or to assign a new customer to any of these classes. Customers are not exclusively assigned to one cluster, but they may belong to several clusters to different degrees. This corresponds more to reality, even though the labels of the clusters may suggest that the clusters are highly homogeneous with respect to the elements in them. Since the segmentation process does not lead to a once and for all segmentation ( customers become older, richer or change their position) a repetition of the process may lead to other clusters and to other customers in existing clusters. This dynamic segmentation ( [1]) may also indicate changes in the structure of the customers which otherwise could not be recognized. To avoid misunderstandings it should be mentioned, that the customer segmentation described above is only the core of a larger "Financial Suite" that is used in banks. There is an extensive preprocessing of customer data before segmentation and the results of the segmentation are then used for several purposes, which will be sketched in the next section.

\begin{tabular}{|c|c|c|c|c|c|c|}
\hline & & $\begin{array}{c}\text { Age } \\
\text { Nears] }\end{array}$ & $\begin{array}{l}\text { Income } \\
\text { [S/Manth] }\end{array}$ & $\begin{array}{l}\text { Assets } \\
{[S]}\end{array}$ & $\begin{array}{l}\text { Credit } \\
\text { [S] }\end{array}$ & $\begin{array}{c}\text { Contr_Margin } \\
\text { [SYYear] }\end{array}$ \\
\hline 1. & Senior_Management & 39,47 & $3.871,825$ & $11.096,456$ & $-4.995,550$ & 44,402 \\
\hline 2 & Early_Retired & 59,92 & $1.886,855$ & $8.716,017$ & $-2.422,165$ & 51,125 \\
\hline 3 & Middle_Management & 44,57 & $3.921,299$ & $13.248,014$ & $-92.558,286$ & 546,815 \\
\hline 5 & Pensioner & 76,20 & $1.497,464$ & $21.746,804$ & $-1.804,408$ & 199,306 \\
\hline 6. & Apprentices & 49,50 & $3.654,464$ & $30.242,750$ & $-5.923,847$ & 145,061 \\
\hline 7 & Young_People & 10,07 & 111,358 & $2.485,742$ & -230.935 & 4.772 \\
\hline
\end{tabular}

Figure 2: Centers of the 7 clusters

\subsection{Extending Customer Segmentation to other Areas of Banking}

One of uses could be the allocation of recourses (sales persons, advisors, etc.) to different classes of customers. After they have been assigned to the different segments, profit potentials can be determined for customer classes or single customers. On the basis of these potentials targets for salespersons can be defined, which can then be used to judge the performance of sales persons in different segments. At the same time sales campaigns can be designed and planned for different products or different segments. So far the analysis (segmentation) and the measures derived from them were static, i.e. they investigated structures at a certain point in time. The management of banks is, however, not only interested in static structures but also in the dynamic behavior of customers. They might want to know, for instance, when a customer will leave the bank, long before that happens. Only then counter measure can be taken. This leads to one major complication: Customers are now no longer defined by numerical or other features (at a certain point in time), but customer behavior is defined by vectors or functions over time. Classical fuzzy cluster methods, however, cluster points and not functions. Hence, either those methods have to be modified or new methods have to be developed. It would exceed the scope of this paper to describe in detail these methods and their application to dynamic problems of 


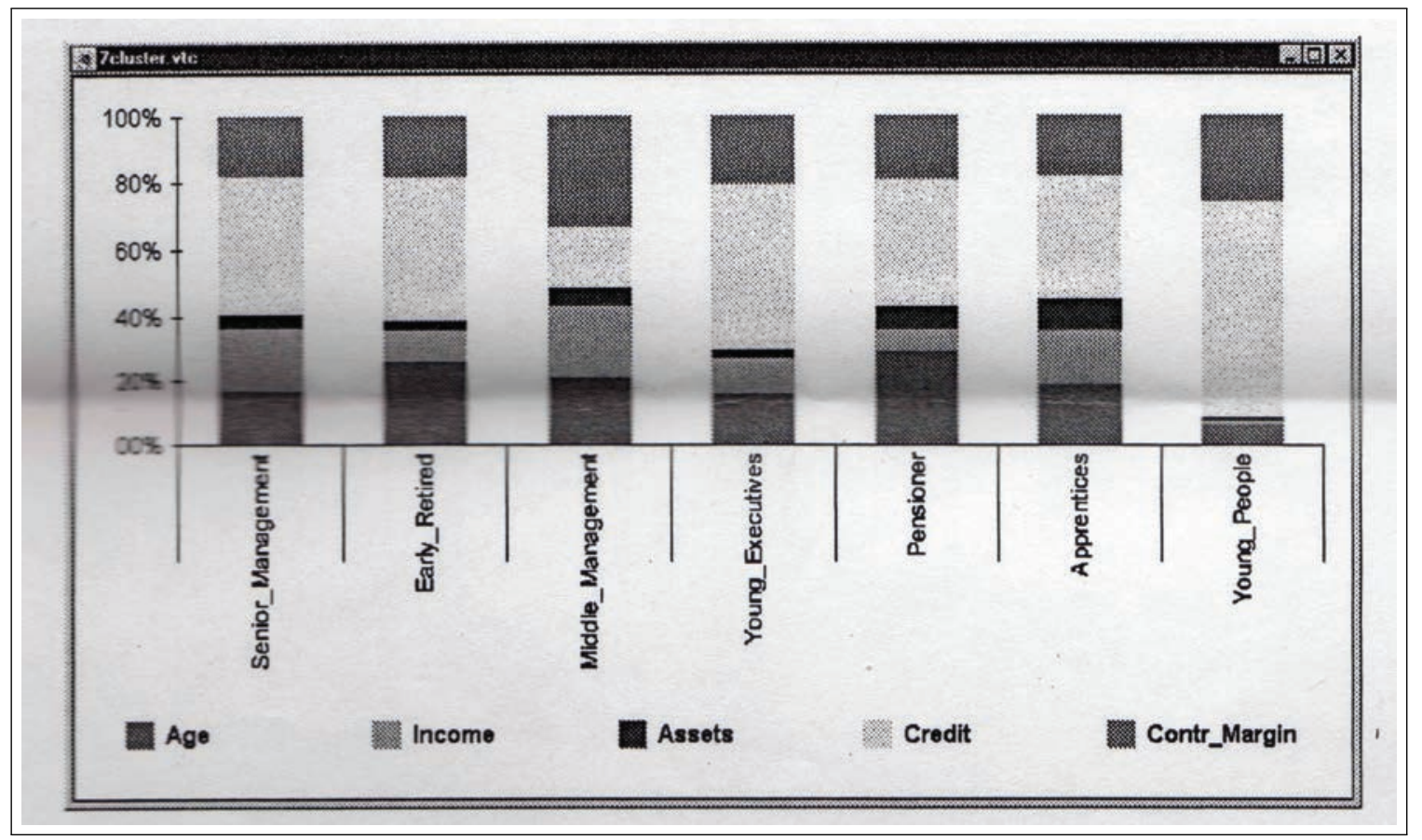

Figure 3: Bar chart of the cluster centers

banks. The interested reader is referred to Angstenberger ( [1]) or Zimmermann ( [30]) for more detailed descriptions and examples of applications.

\section{Conclusions}

Mathematically Fuzzy Set Theory has been developed very far. The application of fuzzy control in engineering problems is also quite accepted and wide spread. In business intelligence, however, applications of fuzzy technology are still rather scarce, even though the original motivation and justification of fuzzy set theory was to model and improve human communication. One reason for this might be, that the appropriate modeling of human e is still difficult and context dependent rather than generic. More contributions of psycho-linguistics in this direction are still desirable. Another reason may be, that the successful application of fuzzy technology into management systems requires the acceptance of this technology by managers. This in turn is strengthened by considering management needs, views, and attitudes already when developing applications in this area. Successful pilot applications my also be useful. In this paper such applications are described in the area of financial management. Technologically fuzzy expert systems as well as fuzzy cluster approaches are used. It would have exceeded the scope of this paper to describe the theoretical bases on which the applications rest in detail. We have rather tried to stress those features which are considered to be important from a manager's point of view. All the applications described are real world applications which have shown to be very successful. We hope to have presented attractive problem solutions which can be used as pilot applications and, hence, increase the application of fuzzy technology in the management area. 


\section{Bibliography}

[1] L. Angstenberger, Dynamic Fuzzy Pattern Recognition with Applications to Finance and Engineering, Kluwer Academic Publishers, Boston, Dodrecht, London, 2001

[2] J.C. Bezdek, Pattern Recognition with fuzzy objective function algorithms, New York, London, 1981

[3] J.C. Bezdek, J.D. Harris, Fuzzy partitions and relations, Fuzzy Sets and Systems 1, 111-127, 1978

[4] B.Bouchon-Meunier, R.R.Yager, L.A.Zadeh (edtrs.) Uncertainty in Intelligent and Information Systems, World Scientific, Singapore, 2000

[5] H. Dishkant, About membership function estimation, Fuzzy Sets and Systems 5, pp.141-147, 1981

[6] D. Dubois, H. Prade, A review of fuzzy set aggregation connectives, Information Science 36, pp.85-121, 1985

[7] Ch. Freksa, Linguistic description of human judgments in expert systems and in the soft sciences, in: M.M. Gupta, E. Sanchez (edtrs), Approximate Reasoning in Decision Analysis, Amsterdam, New York, Oxford, 1982

[8] J.M. Hammerbacher, R.R Yager, The personalization of security:An application of fuzzy set theory, Fuzzy Sets and Systems 5, pp. 1-9, 1981

[9] H.M. Hersh, A. Caramazza, H.H. Brownell, Effects of context on fuzzy membership functions, in:M.M. Gupta et.al.(edtrs) Advances in Fuzzy Set Theory and Applications, Amsterdam, New York, Oxford, 1979

[10] INFORM, fuzzyTECH 5.8, User Manual, available via www.fuzzytech.com, Aachen, 2010

[11] INFORM, RiskShield 4.0, User Manual, available via www.riskshield.com, Aachen, 2011

[12] R.Krishnapuram, J.M.Keller, A possibilistic approach to clustering, IEEE Trans. Fuzzy Systmes 1, 98-110, 1993

[13] E.H. Mamdani, Application of fuzzy logic to approximate reasoning, IEEE Trans.Comput. 26, pp. 1182-1191, 1977

[14] MIT DataEngine Manual 2.1, MIT GmbH, Aachen, 1997

[15] M. Mizumoto, H.-J. Zimmermann, Comparison of fuzzy reasoning methods, Fuzzy Sets and Systems,8, pp.253-283, 1982

[16] A.M. Norwich, L.B. Turksen, A model for the measurement of membership and consequences of its empirical implementation, Fuzzy Sets and Systems 12, pp.1-25, 1984

[17] D. Ruan, A critical study of widely used fuzzy implication operators and the inference rules in fuzzy expert systems, Ph.D. Thesis, Gent 1990

[18] A.Salski, Ecological Modeling and Data Analysis, in: H.-J. Zimmermann (edtr.): Practical Applications of Fuzzy Technologies, Kluwer Academic Publ., Boston 1999, pp.247-266 
[19] U. Thole, H.-J. Zimmermann, P. Zysno, On the suitability of minimum and product operators for the intersection of fuzzy sets, Fuzzy Sets and Systems 2, pp.167-180, 1979

[20] L.A. Zadeh, Fuzzy Sets, Information and Control 8, pp.338-353, 1965

[21] L.A. Zadeh, The concept of a linguistic variable and its application to approximate reasoning, Memorandum ERL-M 411, Berkeley 1973

[22] L.A. Zadeh, Outline of a new approach to the analysis of complex systems and decision processes, IEEE Trans.Syst.Man Cybernet. 3, pp. 28-44, 1973

[23] L.A. Zadeh, The role of fuzzy logic in the management of uncertainty in expert systems, Fuzzy Sets and Systems 11, 199-227, 1983

[24] L.A. Zadeh, A New Frontier in Computation - Computation with Information Described in Natural Language, in: From Natural Language to Soft Computing: New Paradigms in Artificial Intelligence .L.A.Zadeh, Dan Tufis, F.G.Filip, I.Dzitac (Edtrs), Editura Academiei Romane, Bäile Felix (Rom.), 2008

[25] H.-J. Zimmermann, Testability and meaning of mathematical models in social sciences, Mathematical Modelling 1, pp.123-139, 1980

[26] H.-J. Zimmermann, Fuzzy Sets, Decision Making, and Expert Systems, Kluwer, Boston, Dodrecht, Lancaster, 1987

[27] H.-J. Zimmermann, Fuzzy set theory - and inference mechanism, in: G.Mitra (edr.) Mathematical models for decision support, Berlin , Heidelberg 1988

[28] H.-J. Zimmermann, P.Zysno, Latent connectives in human decision making, Fuzzy Sets and Systems 4, pp.37-51, 1980

[29] H.-J. Zimmermann, P. Zysno, Decisions and evaluations by hierarchical aggregation of information, Fuzzy Sets and Systems 10, pp.243-266, 1983

[30] H.-J. Zimmermann, Fuzzy Set Theory and its Applications, fourth edition, Boston, Dodrecht, London, 2001 